\title{
Efectos de la victimización sobre la percepción social de la prisión y las medidas alternativas
}

\section{Miguel Ángel Soria Verde}

Universidad de Barcelona. Facultad de Psicología y Derecho

msoria@ub.edu

\section{Immaculada Armadans Tremolosa}

Universidad de Barcelona. Facultad de Psicología

iarmadans@ub.edu

Recibido: 27-11-2007

Aceptado: 11-06-2008

\section{Resumen}

El artículo presenta una investigación realizada sobre una muestra de 734 residentes en Barcelona ciudad, para conocer la percepción de las personas que han sido víctimas de delitos y las que no sobre la prisión, las medidas alternativas a la prisión (MAP), su función social en la reinserción del delincuente y sus efectos sobre la reducción de la delincuencia. También se analizó el conocimiento sobre las MAP de forma espontánea y sugerida.

Los resultados mostraron cómo las víctimas sostienen una visión más positiva de las MAP frente a la prisión y un mayor conocimiento de las mismas. Por el contrario, no mostraron diferencias en la percepción de la prisión como medio de reinserción o reducción de la delincuencia.

Palabras clave: reinserción social, sociología del delito, delincuencia, actitudes sociales.

Abstract. The effects of victimization on social perception of prison and alternative measures

This research was made in the city of Barcelona with a sample of 734 subjects. It analyzes the vision of victims of crime and no victims about the social effects of prison and alternative measures in the criminal reinsertion and the crime reduction. The second part of this article emphasizes the social degree of knowledge about the alternative measures.

The results show that the victims have a positive vision of alternative measures in front of the traditional prison and a better information and knowledge. But both groups didn't show differences in the vision of prison as a means of rehabilitation or crime reduction.

Key words: social rehabilitation, sociology of crime, delinquency, social attitudes.

\section{Sumario}

1. Introducción

2. Metodología
3. Resultados

4. conclusiones

Referencias bibliográficas 


\section{Introducción}

La aplicación social del nuevo Código Penal en España abrió las puertas a la utilización de las medidas alternativas a la prisión (MAP) como metodología útil en la resocialización de delincuentes y supuso un cambio fundamental en la visión filosófica que apoyaba la intervención social con delincuentes en España (Barona, 1988). Así, frente al criterio "castigo de privación de libertad», se alzó el principio de «justicia distributiva» social, que contemplaba al sujeto como un miembro más de la sociedad donde se hallaba anclado y en gran medida alienado, y defendía la necesidad de estar el máximo de tiempo posible en el seno de un medio social, familiar y laboral normalizado (Chalom y Kousik, 1993).

Un primer grupo de investigaciones se centra en el concepto de "percepción social del riesgo", según el cual las personas perciben una determinada realidad social y reaccionan delante de ésta como si de la misma realidad se tratara, cambiando su conducta (Patterson, 1985) y reforzando actitudes punitivas hacia el delincuente (Hale, 1996; Sprott y Doob, 1997). Pero dicha conclusión no es compartida por diversos estudios, donde se critica que la percepción del riesgo no se corresponde con el riesgo real de la situación o actividad (Espluga, 2004) o bien no encontraron relación entre miedo al delito y deseos de sentencias más severas (Ouimet y Coyle, 1991).

Otro grupo de investigaciones se han centrado en la visión social de la justicia. En Europa, Estados Unidos de América y Canadá, la sociedad tenía una visión negativa de los delincuentes, si bien ello fluctuaba en función de las condiciones económicas y sociales (Melossi, 2000; Sparks, 2000). En España, se criticaba la lentitud y la escasa eficacia del sistema judicial, pero dicha visión era, en gran medida, un estereotipo social, pues sólo un reducido número de los encuestados habían tenido un contacto directo con la justicia (Toharia, 1986). En la misma línea, Ortet y Pérez (1989) estudiaron la actitud de la población universitaria hacia la delincuencia, la población reclusa y el régimen abierto. Los estudiantes de Psicología se mostraron menos conservadores que los de Medicina y más favorables a utilizar la prevención y el tratamiento no punitivo hacia los delincuentes. Posteriormente, los mismos autores (1990) evaluaron la actitud de los equipos de rehabilitación y los funcionarios de prisiones hacia el tratamiento de delincuentes mediante actuaciones socioeducativas o de castigo. Los primeros mostraban una posición favorable hacia la intervención socioeducativa, y los segundos, hacia la punitiva.

Desde una perspectiva actitudinal, Brillon y cols. (1984) analizaron la actitud de la sociedad hacia la rehabilitación de los delincuentes y los centros penitenciarios, observando como las personas más punitivas eran también las más rígidas y conservadoras, y se mostraron más pesimistas respecto a la rehabilitación de los delincuentes, pero no aparecieron diferencias significativas con los no punitivos, en aceptar la aplicación de MAP en determinados tipos penales. Además, estudios sobre la BCS británica (Mattison y Mirrless-Black, 2000) y la Inspección Británica del Crimen (Pennington y Lloyd-Bostock, 1987) mos- 
traron una preferencia pública hacia sentencias dirigidas a la comunidad frente al ingreso penitenciario, así como un elevado nivel de indulgencia en las víctimas y una cuantiosa información respecto a las MAP.

Finalmente, González y Cornejo (1989), partiendo de una exposición fotográfica abierta a la comunidad y elaborada por los internos de una prisión, demostraron la inducción de un cambio de actitud en la comunidad residente en el área de una prisión, al conocer mejor el estilo de vida y los problemas de las personas reclusas.

Siguiendo a Gergen y Gergen (1986) y desde una perspectiva jurídica, las víctimas de actos criminales serían consideradas el colectivo social más reacio y contrario a la utilización de las MAP y, por ello, las defensoras de unas medidas sociales de privación de libertad y de la utilización del castigo como medio compensador del sufrimiento padecido (Dull y Wint, 1997), si bien diversas investigaciones contradicen este planteamiento (Hough y Moxon, 1985; Mattison y Mirrless-Black, 2000).

El presente artículo analiza las diferencias en la visión, el conocimiento y la función social de la prisión tradicional y las MAP entre personas victimizadas y no victimizadas, considerando que las MAP tendrán un coste social bajo, pues su aplicación tendría una visión positiva, al considerarse efectiva sobre los delincuentes. Secundariamente, postulamos que existe una mayor aceptación de la prisión y un mayor conocimiento de las MAP por parte de las personas victimizadas frente a las no victimizadas.

\section{Metodología}

\subsection{Muestra}

Del conjunto de ciudadanos residentes en la ciudad de Barcelona, se seleccionó una muestra de 734 personas mediante un muestreo aleatorio por conglomerados, con dos etapas consecutivas a partir de la lista de calles de la ciudad. Cada conglomerado lo constituía cada página de la lista de calles, posteriormente, dentro de cada conglomerado, todo sujeto mayor de 16 años y residente en las calles seleccionadas se constituía en una unidad del muestreo. El error global máximo es $+/-3,5 \%$ al $50 \%$, con un error alfa del $5 \%$.

Definimos operacionalmente el concepto de víctima como aquella persona que ha sufrido un acto criminal, denunciado o no, durante los doce meses precedentes al estudio.

\subsection{Instrumento de medida}

$\mathrm{Al}$ conjunto de la muestra, se le administró un cuestionario compuesto por 44 preguntas agrupadas en cuatro bloques: datos sociodemográficos de la muestra (edad, sexo, profesión y lugar de residencia), experiencia victimizatoria (haber sido víctima de algún delito durante el último año), conocimiento de las MAP (recuerdo espontáneo o sugerido a partir de su lectura por el entrevista- 
dor) y efectos psicosociales de las MAP (influencia en la reducción del número de delitos y en la reinserción social del delincuente).

En segundo lugar, se estudió la relación existente entre la victimización y los efectos sociales, la reducción de delitos y la capacidad de reinserción social de la prisión y las MAP, mediante un sistema de puntuación escalar de 7 puntos.

\subsection{Procedimiento}

Siguiendo los distintos códigos penales europeos, se seleccionaron nueve MAP para su valoración posterior: arresto de fin de semana; trabajo en beneficio de la comunidad; multa proporcional; penas privativas de derechos; suspensión de pena; reparación civil del daño provocado; sumisión a tratamiento; mediación entre víctima y delincuente, y probation.

Se realizó una entrevista directa y personal por parte de un encuestador entrenado previamente en el domicilio de la calle seleccionada al azar, mediante la aplicación de tablas estadísticas aleatorias al listín telefónico (calles) de Barcelona ciudad. En caso de ausencia del residente, se realizó una segunda visita.

\section{Resultados}

Del conjunto de la muestra analizada, el 9,4\% de los hombres y el 14,4\% de las mujeres habían sido víctimas de algún delito durante el último año. La diferencia entre sexos fue significativa $(5,4 \%)$. Las mujeres habían sido más victimizadas que los hombres.

Las personas víctimas de un delito consideraban que la prisión no reducía el número global de delitos (3,5 puntos) y no reinsertaba (2,1 puntos), de forma similar a las no víctimas (3,8 y 2,4 puntos, respectivamente) (escala máxima de 7 puntos).

Respecto a las MAP, observamos una tasa superior respecto a la prisión. Ambos grupos, víctimas y no víctimas, consideran que inciden en una reducción de los delitos producidos (4,7 puntos de media en las víctimas y 4,3 en las no víctimas), al mismo tiempo, les atribuyen una mayor capacidad de reinserción social (4,5 y 4,3 puntos, respectivamente).

La valoración de ambos grupos, víctimas y no víctimas, sobre los efectos de la prisión y las MAP en reducción de la tasa de delitos fueron coinciden-

Tabla 1. Victimización y efectos sociales de la prisión y las MAP

\begin{tabular}{lccccc}
\hline & \multicolumn{2}{c}{ Prisión } & & \multicolumn{2}{c}{ MAP } \\
\cline { 2 - 3 } \cline { 5 - 6 } & Víctimas & No víctimas & & Víctimas & No víctimas \\
\hline Reducción delitos & 3,5 & 3,8 & & 4,7 & 4,3 \\
Reinserción & 2,1 & 2,4 & & 4,5 & 4,3 \\
\hline
\end{tabular}

Escala de 7 puntos. 
Tabla 2. Victimización y efectos de la prisión sobre la reducción del delito y la reinserción del delincuente $(\%)$

\begin{tabular}{lccccc}
\hline & \multicolumn{2}{c}{ Reducción del delito } & & \multicolumn{2}{c}{ Reinserción } \\
\cline { 2 - 3 } \cline { 5 - 6 } & Víctimas & No víctimas & & Víctimas & No víctimas \\
\hline Mucho & 8,8 & 5,1 & & 49,4 & 39,1 \\
Bastante & 14,3 & 10,3 & & 17,6 & 17,6 \\
Un poco & 12,1 & 14,2 & & 8,8 & 17 \\
Sin cambios & 48,4 & 41 & & 15,4 & 12,8 \\
Bajaría un poco & 9,9 & 16,3 & & 4,4 & 6,7 \\
Bajaría bastante & 4,4 & 8,6 & & 2,2 & 3 \\
Bajaría mucho & 1,1 & 2,2 & & 1,1 & 1,2 \\
Ns/nc & 1,1 & 2,3 & & 49,4 & 39,1 \\
\hline
\end{tabular}

tes, al considerar que la prisión no desempeña ningún papel en el incremento o en el decremento de los delitos. Dicha categoría escalar es seleccionada por el $48,4 \%$ de las víctimas y por el $41 \%$ de las no víctimas.

Respecto a los efectos reinsertadores de la prisión, se observa una disparidad de opiniones, así, el 49,4\% de las víctimas y el 39,1\% de las no víctimas consideran que la prisión no reinserta en absoluto. No obstante, las primeras sólo consideraron el 8,8\% que reinsertaba poco y la tasa se incrementaba hasta el $17 \%$ en el caso de las no víctimas.

El reconocimiento sugerido de las MAP fue superior de forma significativa en las víctimas $(45,9 \%)$ respecto a las no víctimas $(36,2 \%)$, con una diferencia intervictimización del 9,7\%, mientras que el espontáneo se situó en el 34,1\% y el $26,4 \%$, respectivamente, en ambos grupos.

Además, al comparar el recuerdo sugerido con el espontáneo en ambos grupos, observamos una diferencia estadísticamente significativa, cuando el $73,6 \%$ de las personas encuestadas no victimadas son incapaces de citar una sola MAP de forma espontánea, mientras que ello tan sólo sucede en el 65,9\% de las víctimas.

A continuación, se analizaron las posibles diferencias entre los dos grupos y en el reconocimiento sugerido de las MAP. Las más citadas y conocidas por las personas victimizadas fueron el trabajo en beneficio de la comunidad $(62,6 \%)$, la sumisión a tratamiento $(59,3 \%)$ y el arresto de fin de semana $(54,9 \%)$. La más desconocida fue la probation (26,4\%).

Respecto a las personas no victimizadas, las MAP más nombradas fueron la sumisión a tratamiento $(53,3 \%)$, el trabajo en beneficio de la comunidad $(50,4 \%)$ y el arresto de fin de semana $(43,5 \%)$. Al igual que en el otro grupo, la menos conocida resultó ser la probation $(16,2 \%)$.

Así, el trabajo en beneficio de la comunidad fue la primera medida alternativa en ser citada por las víctimas y la sumisión al tratamiento en las no víctimizadas.

En los efectos de las MAP sobre la tasa de delitos y la reinserción del delincuente, ambos grupos no mostraron ninguna diferencia significativa en su opi- 
Tabla 3. Victimización y reconocimiento sugerido de las MAP (\%)

\begin{tabular}{lcc}
\hline & Víctimas & No víctimas \\
\hline Arresto de fin de semana & 54,9 & 43,5 \\
Trabajo de interés general & 62,6 & 50,4 \\
Multa proporcional o días de multa & 39,6 & 28,1 \\
Penas privativas de derechos & 37,4 & 27,8 \\
Condena condicional & 50,5 & 42,5 \\
Reparación civil del daño provocado & 45,1 & 37,2 \\
Sumisión a tratamiento & 59,3 & 53,3 \\
Mediación entre víctima y delincuente & 37,4 & 7,4 \\
Probation & 26,4 & 16,2 \\
\hline
\end{tabular}

nión respecto a la reducción de los delitos por las distintas MAP, sin superar en ningún caso una diferencia intergrupos de 0,2 puntos (escala de 7 puntos). Tanto víctimas como no víctimas consideraron que la MAP más efectiva en la reducción de la criminalidad era la sumisión a tratamiento $(5,5$ y 5,4 puntos, respectivamente) y la multa proporcional como la menos efectiva (4 y 4,1 puntos, respectivamente).

Respecto a la medida más efectiva en la reinserción del delincuente, tampoco aparecen diferencias significativas entre ambos grupos (máximo de 0,2 puntos) y comparten la opinión de considerar la sumisión a tratamiento como la medida más efectiva (5,5 puntos en víctimas y 5,3 en no víctimas) y la menos efectiva, los días de multa (3,6 puntos en ambos grupos).

\section{Conclusiones}

Existe una percepción coincidente entre víctimas y no víctimas respecto a una escasa capacidad de resocialización del delincuente por parte del sistema penitenciario tradicional (media-baja), al igual que en su capacidad para reducir el

Tabla 4. Efectos de las MAP sobre la tasa de delitos y la reinserción del delincuente

\begin{tabular}{lcccccc}
\hline & \multicolumn{2}{c}{ Tasa de delitos } & & \multicolumn{2}{c}{ Reinserción } \\
\cline { 2 - 3 } \cline { 5 - 6 } & Víctimas & No víctimas & & Víctimas & No víctimas \\
\hline Arresto de fin de semana & 4,2 & 4,3 & & 4 & 4,1 \\
Trabajo de interés para la comunidad & 5,3 & 5,1 & & 4,9 & 4,9 \\
Multa proporcional o días de multa & 4 & 4,1 & & 3,6 & 3,6 \\
Penas privativas de derechos & 4,7 & 4,5 & & 4,1 & 3,9 \\
Condena condicional & 4,4 & 4,4 & & 4,1 & 4,2 \\
Reparación civil del daño provocado & 4,5 & 4,3 & & 4 & 3,8 \\
Sumisión a tratamiento & 5,5 & 5,4 & & 5,5 & 5,3 \\
Mediación entre víctima y delincuente & 4,6 & 4,7 & & 4,3 & 4,4 \\
Probation & 5,2 & 5,1 & & 5,1 & 4,9 \\
\hline
\end{tabular}

Escala de 7 puntos. 
delito (baja). Ambos grupos mostraron una mayor confianza en las MAP como mecanismos resocializadores y reductores del delito. Estos resultados contradicen las expectativas jurídicas, pero concuerdan con los estudios previos (Hough y Moxon, 1985; Toharia, 1986; Ortet y Pérez, 1989; Hale, 1996). Contrariamente a la opinión social mayoritariamente extendida (Melossi, 2000), las víctimas no buscarían en el sistema penitenciario una especie de venganza por el padecimiento, al considerar las MAP como más reinsertadoras que la prisión y efectivas contra el delito. Secundariamente, las víctimas intentarían lograr un equilibrio cognitivo mediante la adopción de unos principios de «justicia social distributiva», lo cual genera un cambio actitudinal (González y Cornejo, 1989) y, en consecuencia, debe buscarse una explicación más compleja (Sprott y Doob, 1997).

En segundo lugar, los grupos de víctimas y no víctimas difieren en las tasas de MAP citadas especialmente de forma sugerida a favor de las personas victimizadas. Ello puede deberse al propio proceso de victimización criminal, mediante el cual la víctima intenta restablecer su equilibrio emocional a partir de diversos mecanismos afrontativos. Uno de ellos sería la obtención de una mayor información respecto a los procesos policiales y judiciales, se halle o no legalmente emplazada.

En tercer lugar, los dos grupos también coinciden en la opinión respecto a la función social de las MAP (Sparks, 2000; Mattison y Mirrless-Black, 2000). Consideran que las más útiles son el trabajo en beneficio de la comunidad y la sumisión a tratamiento, tanto en la reinserción como en la reducción de los delitos.

El conjunto de resultados nos alientan a continuar analizando las causas que sostienen una percepción social errónea respecto a un rechazo mayoritario de las MAP por parte de las personas victimizadas, cuando en realidad se produce lo contrario, es decir, una mayor aceptación de las mismas y un rechazo medio al sistema penitenciario tradicional.

\section{Referencias bibliográficas}

BARONA, S. (1988). Prisión provisional y medidas alternativas. Barcelona: Bosch.

BRILlON, Y. y cols. (1984). Les attitudes du public canadien envers les politiques criminelles. Montreal: Université Montreal.

Chalom, M. y Kousik, J. (dirs.) (1993). Violence et déviance à Montréal. Montreal: Liber.

DulL, R. T. y WINT, A. V. N. (1997). "Criminal victimization and its effects on fear of crime and justice attitudes». Journal of Interpersonal Violence, núm. 12, p. 748759.

EsplugA, J. (2004). «Conflictes socioambientals i estudi de la percepció social del risc». Papers: Revista de Sociologia, núm. 72, p. 145-162.

Gergen, K. J. y Gergen, M. M. (1986). Social psychology. Nueva York: Springer. GONZÁlEZ, P. y CORNEJO, J. M. (1989). Actitudes sociales hacia la población penitenciaria. Barcelona: Centre d'Estudis Jurídics i Formació Especialitzada.

HaLE, C. (1996). «Fear of crime: a review of the literature». International Review of Victimology, núm. 4, p. 79-150. 
Hough, M. y Moxon, D. (1985). «Dealing with offenders: popular opinion and the views of victims. Findings from the British Crime Survey». The Howard Journal, núm. 24, p. 160-175.

MatTison, J. y MirRleSS-BLACK, C. (2000). Attitudes to crime and criminal justice: Findings from the 1998 British Crime Survey. Londres: HMSO.

MELOSSI, D. (2000). "Changing representations of the criminal». British Journal of Criminology, núm. 40, p. 296-320.

ORTET, G. y Pérez, J. (1989). «Actitud de estudiantes universitarios hacia la delincuencia». Revista de Estudios Penitenciarios, núm. 241, p. 63-70.

- (1990). «Los profesionales penitenciarios ante las causas, prevención y tratamiento de la delincuencia». Delincuencia/Delinquency, núm. 2(3), p. 233-246.

OUIMET, M. y COYLE, E. (1991). «Fear of crime and sentencing punitiveness: comparing the general public and court practitioners». Canadian Journal of Criminology, núm. 33, p. 149-162.

PATTERSON, A. H. (1985). «Fear of crime and other barriers to the use of public transportation by the elderly». Journal of Architectural and Planning Research, núm. 2, p. 277-288.

Pennington, D. y Lloyd-Bostock, S. (1987). The psychology of sentencing. Oxford: Center for Socio-legal Studies.

Ruiz, J. I.; Pedraza, G.; Rodríguez, L. S. y Alvarado, L. (2004). «Actitudes hacia la delincuencia, normas informales y estresares laborales en empleados de prisiones». Revista Colombiana de Psicología, núm. 13, p. 37-50.

SPARKS, R. (2000). «Penal «austerity»: the doctrine of less eligibility reborn?». En: MatTEWS, R. y FRANCIS, P. Prison 2000: an international perspective on the current state and future of imprisonment. Londres: Macmillan.

SprotT, J. B. y Doob, A. N. (1997). «Fear, victimization, and attitudes to sentencing, the courts and the police». Canadian Journal of Criminology, núm. 39, p. 275291.

ToHARIA, J. J. (1986). «La imagen de la justicia». En: Burillo, F. J. y Clemente, M. (comps.). Psicología social y sistema penal. Madrid: Alianza, p. 105-115. 